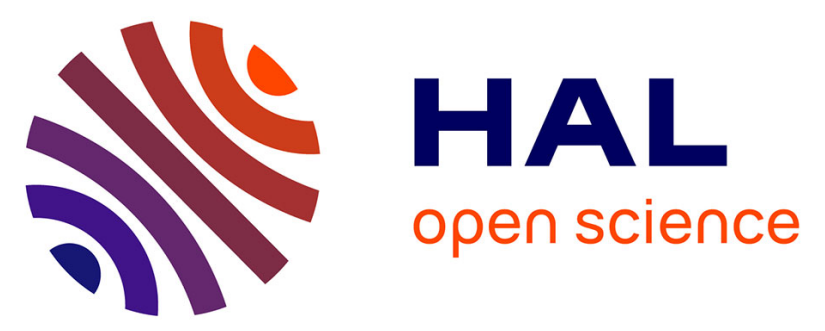

\title{
Blood anti-Müllerian hormone is a possible determinant of recurrent early miscarriage, yet not conclusive in predicting a further miscarriage
}

Estelle Leclercq, Luc de Saint Martin, Caroline Bohec, Marie Thérèse Le Martelot, Sylvie Roche, Zarrin Alavi, Dominique Mottier, Elisabeth Pasquier

\section{To cite this version:}

Estelle Leclercq, Luc de Saint Martin, Caroline Bohec, Marie Thérèse Le Martelot, Sylvie Roche, et al.. Blood anti-Müllerian hormone is a possible determinant of recurrent early miscarriage, yet not conclusive in predicting a further miscarriage. Reproductive BioMedicine Online, 2019, 39, pp.304 311. 10.1016/j.rbmo.2019.04.004 . hal-03488033

\section{HAL Id: hal-03488033 https://hal.science/hal-03488033}

Submitted on 20 Dec 2021

HAL is a multi-disciplinary open access archive for the deposit and dissemination of scientific research documents, whether they are published or not. The documents may come from teaching and research institutions in France or abroad, or from public or private research centers.
L'archive ouverte pluridisciplinaire HAL, est destinée au dépôt et à la diffusion de documents scientifiques de niveau recherche, publiés ou non, émanant des établissements d'enseignement et de recherche français ou étrangers, des laboratoires publics ou privés.

\section{(c) (1) $\$$}

Distributed under a Creative Commons Attribution - NonCommerciall 4.0 International 
1 Blood anti-müllerian hormone is a possible determinant of recurrent early

2 miscarriage, yet not conclusive in predicting a further miscarriage

4 Short title: Blood anti-müllerian hormone level and unexplained recurrent early miscarriage

6 Authors: Estelle Leclercq ${ }^{\mathrm{a}, \mathrm{b}}$, Luc De Saint Martin ${ }^{\mathrm{b}}$, Caroline Bohec ${ }^{\mathrm{c}}$, Marie Thérèse Le

7 Martelot $^{\mathrm{a}}$, Sylvie Roche ${ }^{\mathrm{a}}$, Zarrin Alavi ${ }^{\mathrm{d}}$, Dominique Mottier ${ }^{\mathrm{d}}$, Elisabeth Pasquier ${ }^{\mathrm{b} *}$

$8 \quad{ }^{a}$ Division of Gynaecology, Brest University Hospital, Brest, France

$9 \quad{ }^{\mathrm{b}}$ EA 3878 (GETBO), Department of Internal Medicine and Chest Diseases, Brest University

10 Hospital, Brest, France

$11{ }^{\mathrm{c}}$ Division of Gynaecology, François Mitterand Hospital, Pau, France

12 d INSERM, Centre d'Investigation Clinique - 1412, Brest University Hospital, Brest, France 13

$14{ }^{*}$ Corresponding author

15 E-mail: elisabeth.pasquier@ chu-brest.fr

16

17 


\section{Abstract}

Research Question: Is blood anti-müllerian hormone (AMH) level a strong determinant of unexplained recurrent early miscarriage?

Design: In the first part of the study, AMH levels measured using the ELISA kit of Immunotech* (Beckman-Coutler, France) were compared between 188 all-comer (mostly fertile) women consecutively referred for $\geq 3$ miscarriages in the first trimester of pregnancy and 376 age-matched parous women without pregnancy loss. Cases and controls were previously enrolled in an incident case-control study on thrombophilic mutations. Blood samples were collected $>2$ months after any recognized obstetric event or hormonal treatment. In the second part of the study, a prospective 2-year follow-up of cases was performed.

Results: When considering all women whatever their age, AMH level did not significantly differ between cases and controls $(P=0.152)$. However, in the subgroup $\geq 25$ years old (176 cases vs 358 controls of $\sim 33.5$ years), the cases had significantly lower AMH levels than the controls (median[IR]: $2.8[1.4-4.7]$ vs $3.25[1.7-5.5], P=0.046)$ and the proportion of cases with an $\mathrm{AMH}$ level <1 ng/ml was significantly higher (17.6\% vs $10.6 \%$; OR, 1.80; 95\% CI, [1.073.00], $P=0.028$ ). In regard with the subsequent pregnancy, AMH level was not correlated with either the conception delay or the miscarriage occurrence $(P=0.88)$. However increased age and number of previous miscarriages were significantly predictive of a subsequent miscarriage $(P=0.046$ and $P=0.03$, respectively).

Conclusion: An altered ovarian reserve is a possible determinant of unexplained recurrent early miscarriage. However, AMH blood level predicts neither the delay nor the outcome of a subsequent pregnancy.

Key, words: Recurrent miscarriage, anti-müllerian hormone, ovarian reserve, case-control study, prospective cohort study, fertile women 
43 Key message: Although an altered ovarian reserve might contribute to unexplained recurrent

44 early miscarriage, AMH measurement is less accurate than the civil age or the number of 45 previous losses in prediction of a further loss. 


\section{Introduction}

Recurrent early miscarriage (REM) is currently defined by three or more consecutive losses occurring before 12 weeks' gestation (Delabaere et al, 2014). REM affects one to two percent of couples trying to have children. Standard investigations fail to reveal any well-recognized cause in more than $50 \%$ of the couples (Branch et al, 2010).

Ovarian dysfunction, through either oocyte quality or ovarian hormone production, has been put forward as a possible underlying mechanism of REM. In particular, a reduced ovarian follicle pool is suspected to be associated with REM. Increased maternal age is characterized by a quantitative alteration of the ovarian reserve and is also a well-known determinant of REM (Brigham et al, 1999) suggesting a combined quantitative and qualitative alteration of the ovarian reserve. Nevertheless, although chromosomal aberrations of the abortion product increases with maternal age (Pellestor et al, 2003), the exact correlation between those qualitative alterations and the quantitative ovarian reserve status still remains to be clarified (Plante et al, 2010; Jiang et al, 2018) especially in women aged <35 years old (yo).

In addition to antral follicle count (AFC) by ultrasound, ovarian reserve can be quantitatively assessed by measurement of FSH, inhibin B and AMH blood levels (Broer et al, 2014). AMH blood level is highly correlated with AFC (Hansen et al, 2011). However, discordance was reported. Patient-specific features (e.g. PCOS, age, BMI, altered folliculogenesis or steroidogenesis) may contribute to the differences between measured serum AMH concentrations and AMH values expected from the corresponding AFC, suggesting that AMH might also be a qualitative follicle marker (Alebic et al, 2018). AMH measurement can be easily obtained from a blood sample collected whatever the day in the menstrual cycle. In the female foetus, AMH is secreted by the ovarian granulosa since 36 weeks' gestation. Increase in plasma $\mathrm{AMH}$ concentration reaches a first peak between 7 and 10 yo and a second one between age 20 and 25 yo (Kelsey et al, 2011). Decrease in plasma AMH concentration 
begins around 25 yo and AMH becomes undetectable before menopause (Freeman et al, 2012). Thus, after 25 yo, the AMH blood level appears to be a more reliable marker of the ovarian pool size than the civil age. Moreover, the decline in AMH level precedes the increase in FSH and inhibin levels and can be regarded as an early predictor for progressive reduction of the ovarian reserve. Nevertheless, a recent individual patient data meta-analysis (Depmann et al, 2018), reported only a slight added value of AMH combined with age as a predictor of menopause. Although the authors confirmed that AMH was a significant predictor of time to menopause (especially time to early menopause), an individual prediction of age at menopause was not accurate enough to be routinely used in clinical practice.

In infertile women, studies (Sahu et al, 2010; Tremellen et al, 2010; Tarasconi et al, 2017; Bishop et al, 2017) have assessed whether quantitative markers of the ovarian reserve would predict the occurrence of a miscarriage and a live-birth after intra-uterine insemination or in vitro-fertilization. They reported discrepant results. Although those markers, especially serum $\mathrm{AMH}$ levels, help to predict the intensity of ovarian response to a controlled stimulation, they do not seem to reflect oocyte quality.

Among 348 non selected (mostly fertile) women, age-adjusted AMH levels were retrospectively correlated with reproductive outcomes, no specific AMH-related pattern was suggested (La Marca et al, 2012). In the same way, Zarek et al. did not show any association between AMH level and further pregnancy loss in fertile women with a history of one to two previous losses (Zarek et al, 2016). In contrast, Schumacher et al found that fertile women with very low blood AMH levels $(\leq 0.4 \mathrm{ng} / \mathrm{mL})$ had a 2.3 -fold increased risk of miscarriage compared with women with AMH levels $\geq 1 \mathrm{ng} / \mathrm{mL}$. However, they did not observe an increased risk of miscarriage for women with $\mathrm{AMH}$ levels between 0.4 and $0.7 \mathrm{ng} / \mathrm{mL}$ (Schumacher et al, 2018). This is consistent with the findings in infertile women and further 
supports the controversial role of blood AMH level as an accurate marker of oocyte quality in all-comer fertile women.

What about women with REM? Lower plasma levels of oestradiol and higher plasma levels of FSH at day 3 of the menstrual cycle were reported in women with previous unexplained REM, compared to women with well-identified causes of REM (Trout et al, 2000), suggesting a reduced ovarian reserve. Before our study was designed, only one study had reported the comparison of AMH levels between 34 fertile women with REM and 10 controls. No significant difference was found (Prakash et al, 2006). However, in two recent studies, 70 cases with unexplained REM displayed significantly lower AMH blood levels than those of 70 healthy controls seeking contraception (prospective turkish cohort) (Atasever et al, 2016) or than those of 78 women with explained REM (retrospective study in Austria) (Pils et al, 2016).

Facing the above discrepant reports, we raised the question of whether an altered ovarian reserve was a strong determinant of unexplained REM in all-comer (mostly fertile) women. In the absence of an accurate qualitative marker of the ovarian reserve, we chose to use blood AMH measurements in assessment of ovarian reserve among women with unexplained REM compared with age-matched parous controls. We hypothesized that, unlike AFC, blood AMH could be used not only as a marker of ovarian reserve size but also of ovarian function/oocyte quality. All these women were previously enrolled in our master incident case-control study on thrombophilic mutations (Pasquier et al, 2009). Additionally, we planned to study the prospective outcome of the subsequent pregnancy, according to the blood AMH level, in the cases. 
117 Materials and methods

118 Study design

119 The study was organized in two parts: an incident cases-control study and a prospective 2-

120 year follow-up of cases (Figure 1).

121 Incident case-control study

122 A 1:2 paired incident case-control study was set up to compare blood AMH levels between women referred for unexplained REM defined as 3 or more miscarriages in the first trimester of pregnancy (cases) and control women paired with respect to age, to within 1 year, with cases. The blood samples were collected randomly throughout the menstrual cycle, at least 2 months after any recognized obstetric event or hormonal treatment.

127 Both women groups were enrolled from February 2003 to 2008 in our master study on thrombophilic mutations (Pasquier et al, 2009). In this previous incident case-control study on thrombophilic mutations, pregnancy loss was defined as two or more unexplained consecutive miscarriages with the same partner at or before 21 weeks of gestation, or at least one

131 unexplained pregnancy loss after 21 weeks of gestation. Couples were seen in consultation by 132 one of the physician investigators who provided both complete oral and written information.

133 All couples gave their informed consent. Then the investigator conducted a standard 134 questionnaire survey and venous blood sampling.

135 This ancillary study on AMH involves studying the whole subgroup of women with three or 136 more previous miscarriages in the first trimester of pregnancy (REM). Two controls, the most 137 comparable in regard with age, were selected for each case.

\section{Prospective 2-year follow-up of cases}

139 All the women, who participated in our case-control study on thrombophilic mutations 140 (incident case-control study), were secondly enrolled in a prospective cohort follow-up study. 
141 They were contacted by phone to collect new obstetric and medical events. In addition, all

142 documents related to medical visits and hospitalizations were retrieved.

143 We planned to seek prospectively a correlation between AMH levels and occurrence of a

144 miscarriage in the subsequent pregnancy of REM cases (i.e. from the incident case-control

145 study) during the first 2 years of follow-up.

146

147 Cases

148 All women cases, 18 to 45 yo, were from Brittany. They were sent by their obstetricians and

149 were consecutively seen in internal medicine consultation for a history of unexplained REM.

150 Most cases were not followed-up by a fertility care center. REM was defined as 3 or more

151 miscarriages in the first trimester of pregnancy. Exclusion criteria consisted of: a maternal or

152 paternal carrier of a structural chromosomal rearrangement, maternal antiphospholipid

153 antibodies or any anatomical abnormality likely to be responsible for REM.

154

155 Controls

156 The controls were recruited during the same period, among women 18 to 45 yo registered on

157 the local electoral lists. Women were potentially eligible if they had given birth to at least one

158 living child. Exclusion criteria included pregnancy loss and preclinical miscarriages.

159 Use of assisted reproductive technology (ART) and polycystic ovary syndrome (PCOS) were

160 not considered as exclusion criteria for both cases and controls. Women were diagnosed as

161 "definite PCOS" when they met the internationally recommended criteria (Rotterdam

162 ESHRE/ASRM-Sponsored PCOS Consensus Workshop Group, 2004). 
164 Samples

$1655 \mathrm{ml}$ of blood was collected by venipuncture into a SST gel tube (Vacutainer, Becton-

166 Dickinson) and then centrifuged at $3500 \mathrm{rpm}$ for 15 minutes at room temperature. Then the

167 serum was divided into aliquots of $\sim 300 \mu \mathrm{l}$ and stored at $-80^{\circ} \mathrm{C}$ until use.

168

169

\section{AMH measurements}

170 In 2009, for both cases and controls, the measurement of AMH blood level was

171 simultaneously carried out on $300 \mu \mathrm{l}$ of serum bank using the ELISA kit of Immunotech*

172 (first generation test, Beckman-Coutler, France).

173

174 Statistical Analysis

175 Statistical analysis was performed using SPSS Statistics v.19.

176 Firstly, we planned to compare AMH blood levels between cases and controls regardless of

177 age. Noteworthy, no sample size calculation was done: before our study was designed, only

178 one study had reported similar AMH levels between 34 REM women and 10 controls

179 (Prakash et al, 2006).

180 A model of AMH concentration from conception to menopause clearly demonstrated an

181 AMH peak at 25 yo, after a rise and before a decline in AMH concentrations (Kelsey et al,

182 2011). Consequently, we planned to check whether the distribution of AMH blood levels

183 according to age also depicted a peak $\sim 25$ yo. This would allow to do separate analyses in the

184 subgroups of cases and controls $<25$ yo and $\geq 25$ yo, when the sample size was sufficient (i.e.

$185>30)$. 
186 Student's t-test and chi-square test were used for continuous variables and categorical

187 variables, respectively. The odds ratio (OR) was calculated using logistical regression. $P<0.05$

188 was considered statistically significant. The ORs were adjusted according to predefined

189 variables (e.g. maternal age, smoking, $\mathrm{BMI})$ and potential confounding variables $(\mathrm{P}<0.2)$ as

190 follows: number of previous live births, age at the first obstetrical event, use of ART and

191 definite PCOS.

192 Lastly, for each case to undergo natural conception, we studied, the delay and outcome of the

193 subsequent pregnancy (early miscarriage or not) occurring during the first 2 years of follow-

194 up according to AMH level by cox and logistic regression, respectively. Adjustment for age

195 and number of previous losses was performed.

196 The study was approved by the local ethics committee, the CPP of Brest University Hospital

197 (CCP Ouest-336).

198 
The AMH blood level was available for the 188 cases with REM and 376 age-matched

201 controls, from our previous study on thrombophilic mutations (Pasquier et al, 2009). Except

202 for parity, the baseline characteristics were similar between cases and controls (Table 1).

203 Noteworthy, the age at inclusion, i.e. when blood was collected, was normally distributed and

204 not statistically different between cases and controls ( $32.9 \pm 5$ vs. $33 \pm 4.6$ years, $P=0.8)$. The

205 age at the first obstetrical event (loss or live birth) was slightly but significantly higher in

206 cases compared to controls $(27.4 \pm 5.2$ vs. $26.1 \pm 3.6$ years, $\mathrm{p}=0.003)$. The cases experienced

207 between 3 and 17 early miscarriages. Of the 188 cases, $90(47.8 \%)$ had previously given a live

208 birth. There was no significant difference in delivery term and weight at birth in comparison

209 to the controls.

210 Only 9 cases and 13 controls underwent ART. Main indications for ART were male infertility

211 (for 3 cases and 3 controls), fallopian tube impairment (for 1 case and 2 controls), and 212 endometriosis (for 2 controls). Noteworthy, at least one previous conception without ART 213 was recorded for all the 9 cases vs. only 4 controls. Furthermore, 6 cases and 13 controls had 214 a "definite PCOS".

215 The distribution of AMH levels in the serum of cases and parous control women was not 216 normal, ranging from 0.1 to $18.8 \mathrm{ng} / \mathrm{ml}$ (median[IR]: 2.9[1.5-4.9]) for the cases and from 0.1 217 to $21.5 \mathrm{ng} / \mathrm{ml}$ (median[IR]: 3[1.7-5.5]) for the controls. AMH levels did not significantly 218 differ between cases and controls $(P=0.152)$. Among the 188 cases $31(16.5 \%)$ had an AMH 219 level $<1 \mathrm{ng} / \mathrm{ml}$ in comparison to 40 (10.6\%) controls among 376 (OR, 1.66; 95\% CI, [1.00220 2.75], $\mathrm{p}=0.059$ ). Adjusting for maternal age, age at the first obstetrical event, smoking, BMI 221 and parity did not change the results. 
222 Exclusion of the women with "definite PCOS" from the analysis did not change the results.

223 Given that the estimated prevalence of PCOS is around 15\%, and that the first generation test 224 of Beckman-Coutler is known to give higher values than the other tests available then, we 225 looked for the 85 percentile $(>6.87 \mathrm{ng} / \mathrm{ml})$ of the distribution of AMH levels in our 574 226 women. 55 controls and 28 cases had AMH levels above the 85 percentile. Although the 227 ovarian morphology was often lacking, especially for controls, most of these women (45 228 controls and 21 cases) had no clinical evidence of PCOS. Exclusion of these women from the 229 analysis did not change the results.

230 Among cases, AMH levels were significantly lower in the 6 cases who underwent ART 231 compared to the other cases $(P=0.040)$. Among controls, the AMH levels were not different in 232 women who underwent ART compared to the other controls $(P=0.32)$. As expected, after 233 exclusion of these ART women from analysis, AMH levels did not still differ between cases 234 and controls $(P=0.34)$.

\section{Subgroup analyses}

237 The distribution of AMH levels according to age is shown in Figure 2 and demonstrated a 238 peak at 25 yo in agreement with the data reported by Kelsey et al (Kelsey et al, 2011).

\section{Subgroup of women $<25$ yo.}

241 Only 12 cases and 18 controls were $<25$ yo (Table 2 ). 
244 The analysis of the female subgroup $\geq 25$ yo (176 cases of $33.5+/-4.4$ years vs 358 controls of $24533.5+/-4.1$ years $)$ showed significantly lower AMH levels in cases than in controls $(P=0.046$, 246 Table 2). Adjusting for maternal age, age at the first obstetrical event, smoking, BMI and 247 parity did not change the results $(P=0.030)$. Moreover, 31 cases $(17.6 \%)$ had an AMH level $248<1 \mathrm{ng} / \mathrm{ml}$ in comparison to $38(10.6 \%)$ controls (OR, 1.80; 95\% CI, [1.07-3.00], $P=0.028)$. 249 Exclusion of "definite PCOS" women or, exclusion of cases $(n=25)$ and controls $(n=55)$ with 250 AMH levels $\geq 85$ percentile, did not change the results ( $P=0.030$ and $P=0.021$, respectively). 251 Moreover, AMH levels were significantly lower in cases compared to controls $(P=0.039)$ 252 when adjusting for "definite PCOS".

253 When excluding the 24 women who underwent ART, the AMH levels remained lower in 254 cases compared to controls and the absolute difference between cases and controls was 255 similar. However, this difference was no longer statistically significant $(P=0.091)$, thus 256 suggesting a loss of statistical power. Indeed, when using another approach to assess the 257 impact of previous ART, i.e. adjusting for this variable, AMH levels were significantly lower 258 in cases compared to controls $(P=0.046)$.

\section{Prospective follow-up}

262 The prospective outcome of a subsequent pregnancy was assessed for the cases $\geq 25$ yo ( $\mathrm{n}=$ 263 176) during the first 2 years of follow-up. Eleven cases were lost to follow-up. A subsequent 264 naturally conceived pregnancy was observed among 119 cases as follows: in 75, 108 and 115 265 cases, after month-6, -12 and -18 months, respectively. Among these 119 cases, 37 suffered a 266 further miscarriage in the first trimester of pregnancy (Figure 1). 
AMH level regarded as a continuous or discrete variable, and set at different cut-offs 268 (quartiles of the whole distribution and $<1 \mathrm{ng} / \mathrm{ml}$ ) was not correlated with the delay to conception through cox regression analysis (Figure 3)). Adjusting for either age, number of previous miscarriages, BMI, tobacco or parity did not 271 change the results.

272 AMH level, regarded as a continuous variable, was not correlated to the occurrence of a 273 miscarriage (OR, 1.01; 95\% CI, [0.88-1.15], $P=0.88)$. Adjusting for maternal age and the 274 number of previous miscarriages did not change the results, although both variables (i.e. age 275 and number of previous miscarriages) were positively and significantly associated with a 276 subsequent miscarriage (OR, 1.11; 95\% CI, [1.002-1.22], $P=0.046$ and OR, 1.51; 95\% CI, 277 [1.04-2.20], $P=0.03$, respectively). Moreover, the association of a subsequent loss with blood 278 AMH levels set at different cut-offs (quartiles of the whole distribution and $<1 \mathrm{ng} / \mathrm{ml}$ ) was not statistically significant (AMH<1ng/ml: OR, 0.94; 95\% CI, [0.32-2.76], $P=0.91$ ).

280 In addition, using spline analyses and smaller subgroup analyses, we did not identify a clinical 281 effect of AMH in any specific age groups.

282 Lastly, some patients were given treatments (progesterone and/or antithrombotic) for 283 pregnancy maintenance. Nevertheless, the occurrence of a subsequent miscarriage was not 284 significantly associated with either progesterone intake or enoxaparin injections ( $P=1$ and $285 P=0.42$, respectively). Noteworthy, progesterone was never introduced during the 286 periconceptional period but always after delayed menses. 


\section{Discussion}

290 Taking into account all women regardless of age, AMH blood levels were not significantly

291 different between the 188 REM cases and the 376 healthy controls $(P=0.152)$.

292 Among the $534 \geq 25$ yo $(33.5+/-4)$ women, we found statistically significantly lower AMH 293 levels in cases compared to controls (median 2.8 vs $3.25 \mathrm{ng} / \mathrm{ml}, P=0,046$ ) and, the proportion 294 of cases with an AMH level $<1 \mathrm{ng} / \mathrm{ml}$ was significantly higher than in controls $(17.6 \%$ vs 295 10.6\%; OR, 1.80; 95\% CI, [1.07-3.00], $P=0.028)$. Exclusion of women with "definite PCOS" 296 or very high levels of AMH did not modify the results. In contrast, exclusion of the 22 ART 297 women among cases and controls had an impact on the statistical significance of the 298 difference in AMH levels. However, this difference remained within the range of before 299 exclusion results suggesting, rather, a loss of statistical power. Indeed, when adjusting for a 300 previous ART, AMH levels remained significantly lower in cases compared to controls 301 ( $P=0.046)$. The small sample size of the subgroup of women $<25$ yo did not allow data 302 interpretation.

303 Regarding the prospective follow-up of cases, it is noteworthy, that AMH levels were not 304 correlated with the occurrence of a subsequent miscarriage in the first 2 years after blood 305 collection, irrespective of the age subgroups (25-35 and $\geq 35$ yo). However, consistent with 306 the literature (Brigham et al, 1999), the two strong determinants of miscarriage, i.e. woman's 307 age and number of previous miscarriages, were positively and significantly associated with a 308 subsequent loss.

309 Thus, among the $\geq 25$ yo women, though clinically less meaningful, the results of our incident

310 case-control study are in agreement with the two more recently reported case-control studies 311 (Atasever et al, 2016; Pils et al, 2016), which showed significantly lower serum AMH levels 312 in cases compared to controls despite smaller samples. In the prospective turkish cohort 
313 (Atasever et al, 2016), the AFC, usually well-correlated with AMH level (Hansen et al, 2011),

314 was not different between cases and controls. This raises the following question: how the 315 AMH level was regarded in their study ? Was the AMH level regarded as a marker of ovarian 316 follicular activity or follicular pool size in those women $29.5 \pm 4.5$ yo (i.e. $<25$ yo for $16 \%$ of 317 them)? A separate analysis of possible correlation between AFC and AMH according to age 318 ( $<25$ and $\geq 25$ yo) would have been attractive in the study by Atasever et al. In the other study 319 (Pils et al, 2016), as the cases were slightly older than the controls (median [IR]: 33[28-38] vs. 33[28-36], $P=0.124$ ) perhaps, adjusting AMH level for age would have been appropriate.

321 In addition to the large sample of cases and controls, the other strength of our study was the 322 bias-free recruitment of controls from the electoral lists in parallel with that of the cases, from 323 the same geographic area and at the same time period. The controls had in average two 324 healthy children, no history of pregnancy loss and were matched with cases by age within 1 325 year. Moreover, to our knowledge, this is the first time that a correlation between blood AMH 326 levels and subsequent pregnancy outcomes was sought in a prospective follow-up of fertile 327 women with REM. One distinctive feature of our study was the characteristics of the enrolled 328 cases: all-comer women (mostly fertile: 96\%) referred to an internal medicine consultation 329 and not to a fertility center. Some limitations of our study come from the design of our master 330 study on thrombophilic mutations. That said, the present work did not exclude infertile 331 women or those with PCOS. However, adjustments for these confounfing variables and sub332 analyses made it possible to avoid those possible biases. Moreover, this study was not 333 designed to assess ovarian reserve using AFC due to ethics consideration for control women. 334 Lastly, the results of AMH blood levels (measured using first generation ELISA Beckman 335 Coulter kit) cannot be compared with those of the literature. Indeed, the elisa kits used for 336 AMH measurements were different across studies (i.e. a second generation ELISA Beckman 
337 Coulter kit (Pils et al, 2016) or an ELISA kit fromYH Biosearch (Atasever et al, 2016) vs. a

338 first generation ELISA Beckman Coulter kit in our study).

339 In brief, significant lower AMH blood levels were observed among women $\geq 25$ yo with 340 unexplained REM, through comparison with age-matched well-selected controls. 341 Nevertheless, the concentrations were most often within the normal range and, regarding the 342 proportion of women with blood $\mathrm{AMH}<1 \mathrm{ng} / \mathrm{ml}$, the OR was less than 2 . Given that in this age 343 subgroup the AMH level is well correlated with the ovarian reserve size, and consistent with 344 other studies (Atasever et al, 2016; Pils et al, 2016) we cannot rule out that a relative lower 345 ovarian reserve could contribute to occurrence of unexplained REM among women $\geq 25$ yo. 346 However, if we had based a sample size calculation on those two recently reported studies we 347 would have enrolled $\sim 70$ cases and controls and probably would not have found any 348 significant association between AMH level and REM. Furthermore, our follow-up results 349 demonstrated that unlike maternal age and number of previous miscarriages, AMH was not a 350 predictor of REM. These findings were consistent with those reported by a very recent 351 retrospective cohort study among women with idiopathic recurrent miscarriage (Pils et al, 352 2019). Nevertheless, the Pils et al study's results could be regarded as less conclusive due to 353 its retrospective design.

354 In conlusion, our study suggests that an altered ovarian reserve should be regarded as one 355 among other determinants of unexplained REM. However, AMH level measurement is not a 356 useful tool in prediction of a subsequent pregnancy outcome in women with previous 357 unexplained REM. 


\section{Acknowledgments}

359 The authors thank the GYNOBS 29 network.

360 This study was conducted with financial support from the French Health Office (PHRC 2002

361 R08.04 and 2010 08-16) and Organon France for a FARO (Fonds d'aide à la recherche

362 Organon). There are no competing interests.

363

364

365 
Alebic MŠ, Stojanovic N, Dewailly D. Discordance between serum anti-Müllerian hormone concentrations and antral follicle counts: not only technical issues. Hum Reprod. 2018 Jun $1 ; 33(6): 1141-1148$.

Atasever M, Soyman Z, Demirel E, Gencdal S, Kelekci S. Diminished ovarian reserve: is it a neglected cause in the assessment of recurrent miscarriage? A cohort study. Fertil Steril 2016;105: 1236-40.

Bishop LA, Richter KS, Patounakis G, Andriani L, Moon K, M.D.,Devine K. Diminished ovarian reserve as measured by means of baseline follicle-stimulating hormone and antral follicle count is not associated with pregnancy loss in younger in vitro fertilization patients. Fertil Steril 2017; 108: 980-987.

Branch DW, Gibson M, Silver RM. Clinical practice. Recurrent miscarriage. N Engl J Med 2010;363: 1740-1747.

Brigham SA, Conlon C, Farquharson RG. A longitudinal study of pregnancy outcome following idiopathic recurrent miscarriage. Hum Reprod 1999;14: 2868-2871.

Broer SL, Broekmans FJM, Laven JSE, Fauser BCJM. Anti-Mullerian hormone: ovarian reserve testing and its potential clinical implications. Hum Reprod Update 2014;20(5):688701.

Delabaere A, Huchon C, Lavoue V, Lejeune V, Iraola E, Nedellec S, et al. [Definition of pregnancy losses: Standardization of terminology from the French National College of Obstetricians and Gynecologists (CNGOF)]. J Gynecol Obstet Biol Reprod (Paris). 2014 Dec;43(10):756-63

Depmann M, Eijkemans MJC, Broer SL, Tehrani FR, Solaymani-Dodaran M, Broekmans FJM, et al. Does AMH relate to timing of menopause? Results of an Individual Patient Data meta-analysis. J Clin Endocrinol Metab 2018.

392 Freeman EW, Sammel MD, Lin H, Gracia CR. Anti-mullerian hormone as a predictor of time to menopause in late reproductive age women. J Clin Endocrinol Metab 2012; 97: 1673-80.

394 Hansen, KR, Hodnett GM, Knowlton N, Craig LB. Correlation of ovarian reserve tests with histologically determined primordial follicle number. Fertil Steril 2011;95: 170-5.

396 Jiang X, Yan J, Sheng Y, Sun M, Cui L, Chen ZJ. Low anti-Müllerian hormone concentration 397 is associated with increased risk of embryonic aneuploidy in women of advanced age. Reprod 398 Biomed Online. 2018 Aug;37(2):178-183

399 Kelsey TW, Wright P, Nelson SM, Anderson RA, Wallace WH. A validated model of serum 400 anti-mullerian hormone from conception to menopause. PLoS One 2011;6: e22024.

401 Kelsey TW, Anderson RA, Wright P, Nelson SM, Wallace WHB. Data-driven assessment of 402 the human ovarian reserve. Mol Hum Reprod 2012; 18: 79-87. 
La Marca A, Spada E, Grisendi V, Argento C, Papaleo E, Milani S, Volpe A. Normal serum anti-Mullerian hormone levels in the general female population and the relationship with reproductive history. Eur J Obstet Gynecol Reprod Biol 2012; 163: 180-4.

Pasquier E, Bohec C, Mottier D, Jaffuel S, Mercier B, Ferec C, Collet M, De Saint Martin L. Inherited thrombophilias and unexplained pregnancy loss: an incident case-control study. J Thromb Haemost 2009; 7: 306-11

Pellestor F, Andréo B, Arnal F, Humeau C, Demaille J. Maternal aging and chromosomal abnormalities: new data drawn from in vitro unfertilized human oocytes. Hum Genet 2003; 112: 195-203.

Pils S, Promberger R, Springer S, Joura E, Ott J. Decreased Ovarian Reserve Predicts Inexplicability of Recurrent Miscarriage? A Retrospective Analysis. PLoS One 2016;11:e0161606.

Pils S, Stepien N, Kurz C, Nouri K, Springer S, Hager M, Promberger R, Ott J. Does antiMullerian hormone predict the outcome of further pregnancies in idiopathic recurrent miscarriage? A retrospective cohort study. Arch Gynecol Obstet. 2019 Jan;299(1):259-265.

Pils S, Stepien N, Kurz C, Nouri K, Springer S, Hager M, Promberger R, Ott J.

Plante BJ, Beamon C, Schmitt CL, Moldenhauer JS, Steiner AZ. Maternal antimullerian hormone levels do not predict fetal aneuploidy. J Assist Reprod Genet 2010;27: 409-14.

Prakash A, Li TC, Laird S, Nargund G, Ledger WL. Absence of follicular phase defect in women with recurrent miscarriage. Fertil Steril 2006;85: 1784-90.

Rotterdam ESHRE/ASRM-Sponsored PCOS Consensus Workshop Group. Revised 2003 consensus on diagnostic criteria and long-term health risks related to polycystic ovary syndrome. Fertil Steril 2004;81: 19-25.

Sahu B, Oztutrk O, Serhal P, Jayaprakasan K. Do ovarian reserve tests predict miscarriage in women undergoing assisted reproduction treatment? Eur J Obstet Gynecol Reprod Biol 2010; 153: 181-4.

Tarasconi B, Tadros T, Ayoubi JM, Belloc S, de Ziegler D, Fanchin R. Serum antimüllerian hormone levels are independently related to miscarriage rates after in vitro fertilizationembryo transfer. Fertil Steril. 2017 Sep;108(3):518-524.

Tremellen K and Kolo M. Serum anti-Mullerian hormone is a useful measure of quantitative ovarian reserve but does not predict the chances of live-birth pregnancy. Aust $\mathrm{N}$ Z J Obstet Gynaecol 2010; 50: 568-72.

Trout SW and Seifer DB. Do women with unexplained recurrent pregnancy loss have higher day 3 serum FSH and estradiol values? Fertil Steril 2000; 74: 335-7.

Zarek SM, Mitchell EM, Sjaarda LA, Mumford SL, Silver RM, Stanford JB, et al. Antimüllerian hormone and pregnancy loss from the Effects of Aspirin in Gestation and Reproduction trial. Fertil Steril 2016; 105: 946-952. 
442

443

444 


\begin{tabular}{|c|c|c|c|}
\hline & CASES & CONTROLS & \\
\hline $\mathbf{N}$ & 188 & 376 & \\
\hline $\begin{array}{l}\text { Age, } y(\text { mean } \pm \text { SD }) \\
\text { Median }[\text { min-max] } \\
\geq 25 y, n(\%)\end{array}$ & $\begin{array}{c}32.9 \pm 5.0 \\
32.9[18.8-44.8] \\
176(93.6)\end{array}$ & $\begin{array}{c}33.0 \pm 4.6 \\
32.6[18.5-44.7] \\
358(95.2)\end{array}$ & 0.788 \\
\hline Smoking, pack-y $($ mean \pm SD $)$ & $4.8 \pm 6.0$ & $4.4 \pm 6.0$ & 0.551 \\
\hline Body mass index, kg/m2 (mean $\pm \mathrm{SD})$ & $23.0 \pm 4.15$ & $22.8 \pm 4.5$ & 0.603 \\
\hline Menstrual cycle, $d(m e a n \pm S D)$ & $28.5 \pm 3.0$ & $28.8 \pm 3.3$ & 0.342 \\
\hline Previous live birth, n (median [min-max]) & $0[0-4]$ & $2[1-6]$ & $<0.001$ \\
\hline Age at the first obstetrical event, $y($ mean \pm SD) & $27.4 \pm 5.2$ & $26.1 \pm 3.6$ & 0.003 \\
\hline $\begin{array}{r}\text { Assisted Procreation Technology, } n(\%) \\
\text { Intrauterine inseminations, } n \\
\text { In vitro fertilization, } n\end{array}$ & $9(4.8)$ & $13(3.5)$ & 0.442 \\
\hline Polycystic Ovarian Syndrome, n (\%) & $6(3.2)$ & $13(3.5)$ & 0.869 \\
\hline
\end{tabular}

446

447 Table 1. Baseline characteristics of cases and controls. 


\section{Women aged $\geq 25$ years}

\begin{tabular}{|c|c|c|c|}
\hline & CASES & CONTROLS & \\
\hline $\mathbf{N}$ & 176 & 358 & $P$ value \\
\hline $\begin{array}{l}\text { Age, y }(\text { mean } \pm \text { SD }) \\
\text { Median [min-max] }\end{array}$ & $\begin{array}{c}33.5 \pm 4.4 \\
33.3[25-44.8]\end{array}$ & $\begin{array}{c}33.5 \pm 4.1 \\
33.3[25-44.7]\end{array}$ & 0.914 \\
\hline $\begin{array}{l}\text { AMH, ng/ml }(\text { mean } \pm \text { SD }) \\
\text { Median [IR] }\end{array}$ & $\begin{array}{c}3.5+/-3.0 \\
2.8[1.4-4.7]\end{array}$ & $\begin{array}{c}4.1+/-3.4 \mathrm{ng} / \mathrm{ml} \\
3.25[1.7-5.5]\end{array}$ & 0.046 \\
\hline$<1 n g / m l, n(\%)$ & $31(17.6 \%)$ & $38(10.6 \%)$ & 0.028 \\
\hline
\end{tabular}

\begin{tabular}{|l|c|c|} 
& CASES & CONTROLS \\
\hline $\mathbf{N}$ & 12 & 18 \\
\hline Age, $\mathbf{y}(\mathbf{m e a n} \pm$ SD) & $23.0 \pm 2.0$ & $22.7 \pm 2.0$ \\
Median [min-max] & $23.7[18.8-24.9]$ & $23.3[18.5-24.9]$ \\
\hline AMH, ng/ml (mean \pm SD) & $5.0+/-2.25$ & $2.1+/-0.9$ \\
Median [IR] & $5.75[2.75-7.15]$ & $2.3[1.3-2.6]$ \\
\hline
\end{tabular}

451 Table 2. Subgroup results according to age $\geq \mathbf{2 5}$ or $<\mathbf{2 5}$ years

452 Interquartile Ranges: IR 


\section{Figure 1. Flowchart.}

455 Cases and controls enrolled in the incident 1:2 case-control study; subgroups according to 456 age; cases enrolled in the prospective 2-year follow-up with outcome of the first further 457 pregnancy.

458 W: Woman, ART: assisted reproductive technology

459 
460 Figure 2: Distribution of AMH blood levels (ng/ml) according to age (y) among cases and 461 controls

462 
463 Figure 3: 1 -survival curves of the cases aged $\geq 25$ years, according to quartiles of AMH 464 distribution (quartile $1: \leq 1.475,1.475$ <quartile $2 \leq 2.85,2.85<$ quartile $3 \leq 4.825 \mathrm{ng} / \mathrm{ml}$, quartile 465 4>4.825, unit: ng/ml). Cox regression, global $P=0,954$ 466 
INCIDENT 1:2 AGE-MATCHED CASE-CONTROL STUDY
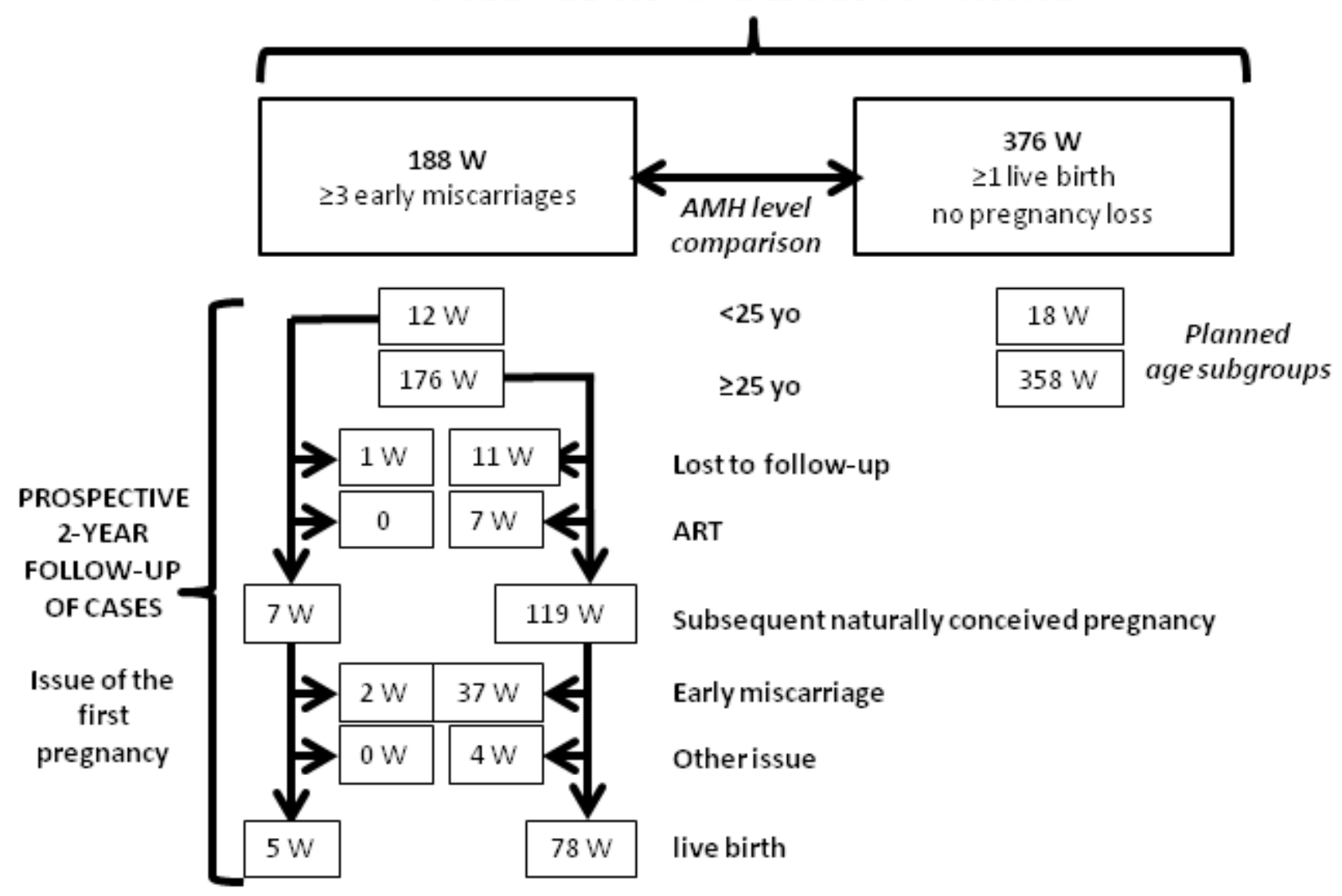


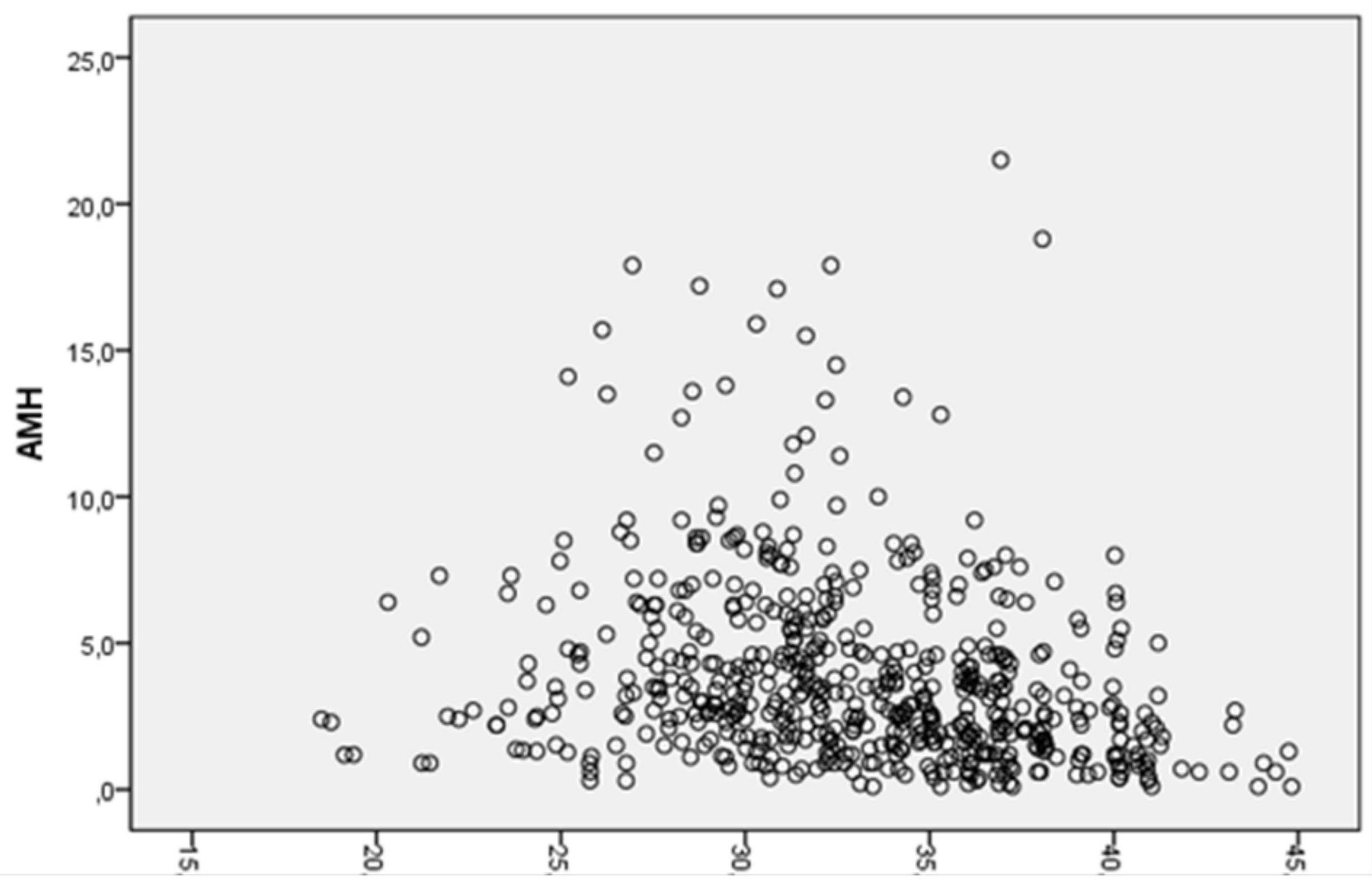




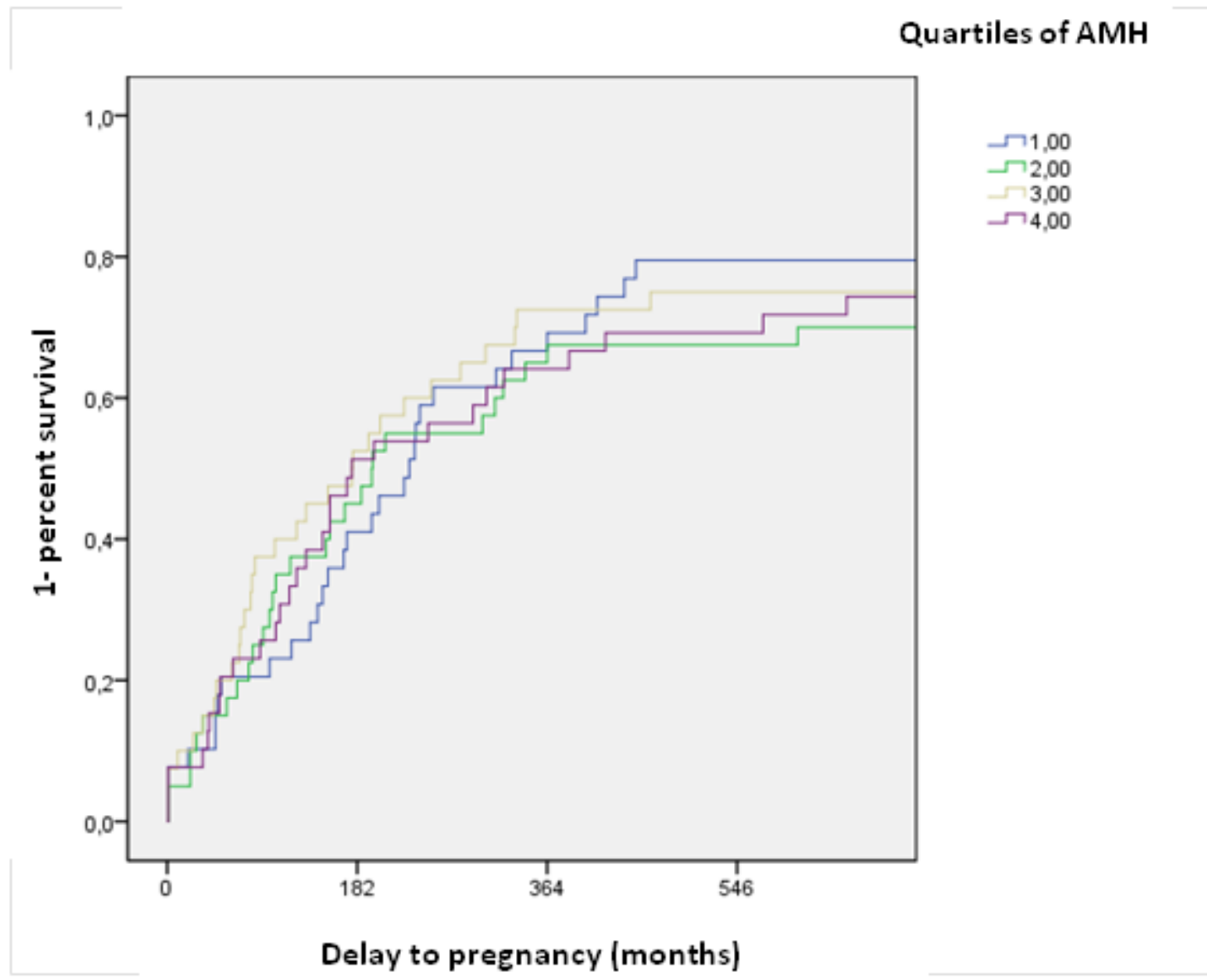

